(C2013 IEEE. Personal use of this material is permitted. Permission from IEEE must be obtained for all other users, including reprinting/ republishing this material for advertising or promotional purposes, creating new collective works for resale or redistribution to servers or lists, or reuse of any copyrighted components of this work in other works. 


\title{
Parallel-Data Reconstruction for Limited Views Tomography Sensors by Sinusoidal Hough Transform
}

\author{
Jose A. Cantoral-Ceballos, Student Member, IEEE, and Krikor B. Ozanyan, Senior Member, IEEE
}

\begin{abstract}
Physical limitations found in industrial environments often restrain imaging for Process Tomography. When information is collected from sparse sensors, the acquired data is limited in terms of radial and angular sampling of the imaged slice. To overcome this problem, we demonstrate in hardware an efficient solution, based on the parallel implementation of the Sinogram Recovery Algorithm (SRA) for limited views, in its variant based on the calculation of the coordinates of the center of mass (CoMs) of the Subject Under Test (SUT), rather than performing the complete sinogram restoration. By introducing a modification in the existing SRA, we achieve high parallelization of each stage, making it ideal for implementation in hardware accelerated systems such as Field Programmable Gate Arrays (FPGAs). The potential to parallelize the SRA is first studied in MATLAB by processing all data projections concurrently and verifying performance by the match between the results from the parallel and sequential implementations. Further, the algorithm is coded in Very High Speed Integrated Circuits Hardware Description Language (VHDL), implemented and tested onto a Xilinx Virtex 6 board. We report speed-ups of between 3 and 4 orders of magnitude whereas the errors in CoMs' coordinates are reduced.
\end{abstract}

Index Terms - FPGA, Hough transform, parallel algorithm, sinogram recovery, tomography, VHDL.

\section{INTRODUCTION}

$\mathrm{D}_{\mathrm{r}}^{\mathrm{s}}$ ue to the limited access often characteristic for Industrial Process Tomography (IPT), using traditional image reconstruction techniques such as Filtered Back-Projection (FBP) can lead to severe artefacts and thus to images which are not useful or even misleading. To combat this limitation different methods have been proposed, based on transformation techniques [1], [2], iterative algorithms using $a$ priori knowledge of the scene [3], [4] and, more recently, 2-D wavelet transformation [5]. However, in spite of some encouraging results, in general these methods are not suited for a severe scarcity in the number of measurements and/or entail high computational complexity compromising their

Manuscript received XXXX X, 20XX. J.A.C.-C. would like to thank the National Council for Science and Technology (CONACyT) of Mexico for PhD studentship. This is an expanded paper from the IEEE SENSORS 2011 Conference.

The authors are with the school of Electrical and Electronic Engineering, University of Manchester, Manchester, United Kingdom (e-mail: k.ozanyan@manchester.ac.uk).

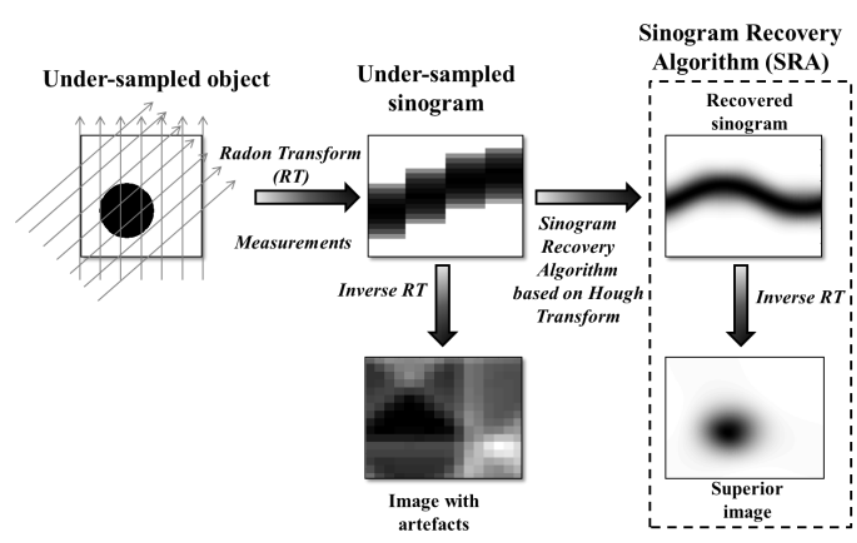

Fig. 1. Sinogram Recovery Algorithm (SRA) for under-sampled sinograms. RT denotes Radon Transform, which is the standard image reconstruction approach for hard-field Tomography sensors.

usability in most real-time scenarios. To address these limitations in IPT sensing and imaging, we focus on the Sinogram Recovery Algorithm (SRA) (see Fig. 1) based on the Sinusoidal Hough Transform (SHT) [6]. The essence of SRA is to enhance the sparsely sampled sinogram allowing a superior quality image reconstruction by standard data inversion. The SRA has been shown to generate satisfactory results from as little as 32 path integrals (sensing "beams") in Photonic Guided Path Tomography (PGPT) applications [7],[8], which is a strong motivation to test the algorithm in different applications with similar sensor geometries [9].

However, the SRA is a computationally expensive algorithm performing calculations of several trigonometric functions which complicate its utilization in an IPT system. Furthermore, previous to this work, the algorithm had only been applied sequentially [6], [8], limiting its execution time and preventing its applications in real-time. In this work, the potential for parallelization of the SRA is identified and realized. A modified parallel algorithm is presented, which significantly reduces the computational requirements, allowing embedded implementation. We target such an embedded system, incorporating the Tomography sensor, performing efficient data acquisition, processing and real-time imaging, as well as the delivery of reconstructed images or signals for control circuits in a parallel multi-processor system based on Field Programmable Gate Arrays (FPGAs). Multichannel FPGA data acquisition systems performing 
simultaneous signal processing (by digital lock-in detection) in 32 independent channels for IPT have already been demonstrated [10]. This puts at the forefront the need to extend the parallel approach further into data processing and image reconstruction.

For the purpose of this work, the different blocks of the SRA were analyzed and possibilities for parallelization were identified. It was recognized that for most IPT applications the relative position of the constituent objects can take prevalence over fine border information. This is because the spatial dynamics of the Subject Under Test (SUT) can be identified from the computations of the coordinates of the centers of mass (CoMs) only.

For the initial stages of this work, different composite phantoms were generated and the individual datasets of the forward Radon transform (RT) were calculated from four angular projections, yielding a severely under-sampled sinogram. The data were further processed with parallel CoM algorithm (PCoMA) independently and concurrently in MATLAB, using standard desktop hardware. The results matched those obtained in the sequential version, thus verifying the correct functioning of the bulk of the parallel algorithm.

The PCoMA was then studied and refined further, to achieve finer grain parallelism. This produced a significant reduction in the complexity of the original SRA. Given the high degree of parallelism in this new approach, implementation and testing were carried out on FPGAs. The results show up to 4 orders reduction in the changes of the execution time and a measurable decrease in the error of the calculated CoM coordinates of the objects contained in the SUT.

\section{SinOGRAm RECOVERY ALGORITHM}

As opposed to medical tomography where structural details are the main priority, most IPT applications are characterized by diffuse distributions and boundaries in which the detection of the time evolution of the CoM of the constituent objects is sufficient to understand and monitor the dynamics of the scene. This changes the emphasis from achieving a sinogram recovery followed by a full image reconstruction to providing means for real-time CoM calculation and tracking by means of hardware acceleration. It is worth noting here that compared to images, calculated CoMs are much easier to utilize with circuits which control the behavior of the SUT. Consequently, the focus of this work is to assess the potential for parallelization and hardware implementation.

As explained in [6], [8], the SRA comprises three main stages, reduction to CoM, SHT and estimation of projections, which are described below. A comprehensive explanation of the individual stages of the SRA is available in [6], [8].

\section{A. Reduction to CoM form}

The algorithm identifies the data bounded within limits of the projection supports $t_{+}\left(\theta_{i}\right)$ and $t_{-}\left(\theta_{i}\right)$ [2] at each angular projection $\theta_{i}$ (see Fig. 2), where $t$ is enumerating the equidistant parallel line integrals (the displacement coordinate

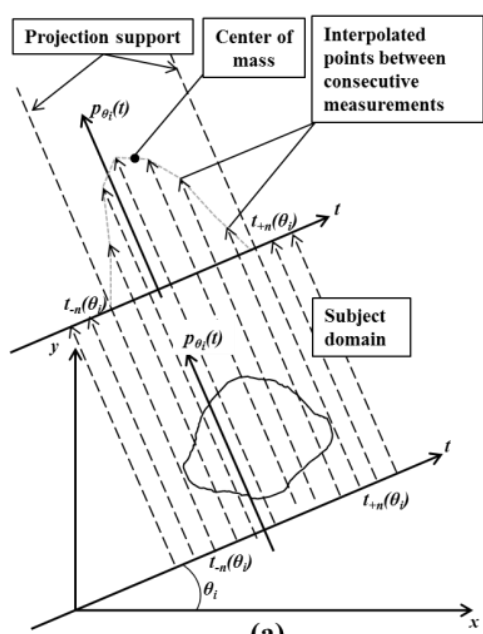

(a)

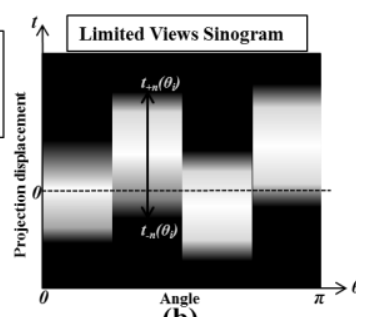

Angle

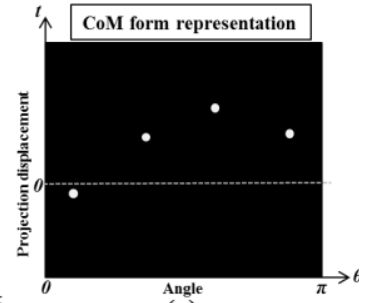

(c)
Fig. 2. a) The support of the projection $p_{\theta i}(t)$ at angle $\theta_{i}$ is determined by that of the subject for each constituent object $n$, which is also represented in b) the Limited Views Sinogram, where it is seen that the support of each angular projection is confined between the same points of the subject $t_{-n}\left(\theta_{i}\right)$ and $t_{+n}\left(\theta_{i}\right)$. Each angular projections is interpolated as shown in a) and then reduced to c) CoM representation. It should be noted that the figure is only for illustration purposes and does not entail a real subject or RT.

in Radon space). Interpolation along $t$ is required because each individual angular dataset is sparsely sampled.

Once the data has been interpolated, the CoM is calculated using the following expression:

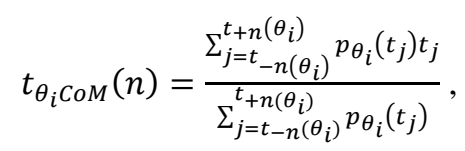

where $\theta_{i}$ represents the different angles of the projections; $n$ enumerates the objects found in each angular projection; $t_{j}$ are the discrete elements of the projection displacement $t ; p_{\theta i}\left(t_{j}\right)$ is the individual value at $t_{j}$ of the interpolated projection $p_{\theta i}(t)$ for angle $\theta_{i}$. Finally, $t_{+n}\left(\theta_{i}\right)$ and $t_{-n}\left(\theta_{i}\right)$ denote the support limits of the $n$th possible object in the projection at angle $\theta_{i}$ as shown in Fig. 2.

After this stage, the sinogram is represented in CoM form at each angular projection; however, it is still angularly underpopulated. The point spread function of the RT is a half-period sinusoid [6], [8], therefore identifying the sinusoidal variation of the CoM position as a function of angle from a small number of points allows SRA to yield unique CoM coordinates.

\section{B. Sinusoidal Hough Transform}

This is the core section of the algorithm. The already calculated sinogram in its CoM form is used as the input to this stage. The SHT converts the CoM pixels into straight lines, defined by the SHT equation [8]:

$$
y=-\frac{\cos \theta_{i}}{\sin \theta_{i}} x+\frac{{ }_{t_{\theta_{i} \operatorname{coM}}(n)}}{\sin \theta_{i}},
$$


where $t_{\theta i C o M}(n)$ is the output of (1) and represents the CoM form of the $n$th object found at angular projection $\theta_{i}$.

The output, after the SHT has been applied, is a twodimensional $x-y$ accumulator, whose peaks represent the predominant sinusoids in the RT.

\section{Estimation of projections}

This final stage is applied if full image reconstruction is required and uses the coordinates of the peaks identified in the previous stage to recover the complete forward RT to a certain degree of approximation. The sinusoids of the predominant objects are calculated for all angles. For this, a particular prior function is assumed to model the contribution of each object [8]. This is followed by the application of standard reconstruction techniques such as Filtered Back-Projection (FBP).

\section{PARALLELIZING THE SRA: MATLAB Results}

Although the SRA has proved to be a good option for imaging from severe under-sampled data, difficulties to implement it in real-time imaging applications arise mostly from the computational cost of the SHT core and postprocessing tasks [6]. This tightens further the typical bottleneck imposed by the image reconstruction stage of such a system. At the same time, there is an important motivation to pursue the computation of the SRA with a view to the development of a complete embedded system capable of performing data acquisition, processing and imaging from sparse data. Thus, exploiting the parallelization potential of the SRA and hardware acceleration are of strong relevance.

In our particular case of tomography applications, parallelization can be achieved from two perspectives: a) coarse-grained parallelism by processing each angular projection independently and b) fine-grained parallelism by treating the sinogram pixel-wise for concurrent pixel processing, in a manner similar to parallelization of the linear Hough Transform [11]-[14]. The approach followed in this work combines both perspectives in a single implementation, using the same algorithm for different sets of data; i.e. the Single Instruction Multiple Data model.

The key element to implement a parallel embodiment of the SRA is to identify data dependencies. The bulk of the SHTbased algorithm cannot be executed before completing the pre-processing, i.e. the CoMs for each angular projection need to be computed before the SHT is applied (pixel-wise) on the set of CoMs. However, each individual angular projection dataset is independent of the others, allowing their simultaneous computation not only for the SHT part, but also for the calculation of their CoM form, as explained further.

In the reduction to CoM-form stage, parallelism is achieved by computing all angular projections simultaneously. The initial interpolation for each dataset is run concurrently, followed by parallel reduction to CoM form. Interestingly, although these two subsections of the initial stage appear to be inherently sequential, in section IV we describe an alternative method in which they can be computed in parallel when implemented in hardware.
Fine-grained parallelism is achieved during the SHT stage. At that point, all CoM-form pixels computed in the previous stage are processed concurrently, leading to the simultaneous generation and detection of peaks in the accumulator. Finally, the third stage of the algorithm can be executed to recover the sinogram trace for each detected object.

To verify the performance of the parallel CoM algorithms, they were tested within the wider framework of a parallel SRA (PSRA) in MATLAB by generating different phantoms and processing each angular projection simultaneously. The phantoms were created to account for the diverse nature of subjects encountered in IPT; varying in number of objects, edge definition and sensor geometry (specifically, the number of beams per projection). This is displayed in the left panels of Fig $3 \mathrm{a}$ and $3 \mathrm{~b}$ showing phantoms representative of subjects with sharp and smooth edges. The RT of each subject was obtained using four angular projections at $0^{\circ}, 45^{\circ}, 90^{\circ}$ and $135^{\circ}$. Then, the reduction to $\mathrm{CoM}$ form for each angular projection was calculated concurrently, followed by simultaneous SHT. The reconstruction was carried out using FBP and a Ram-Lak filter with a Hamming Window to suppress the high frequencies. The MATLAB experiments were executed on shared memory architecture, with the aid of the parallel computing toolbox on a PC with an Intel Core 2 Quad @ $2.83 \mathrm{GHz}$ with 4 GB of RAM. The right panels of Fig. $3 \mathrm{a}$ and $3 \mathrm{~b}$ compare the results for the two generated phantoms after PSRA was applied; each angular projection used 25 beams. It was observed that the differences between the results from SRA and PSRA reconstructions were negligible, since the differences in the CoM coordinates from both implementations are undetectable within the resolution given by MATLAB using double precision floating point.
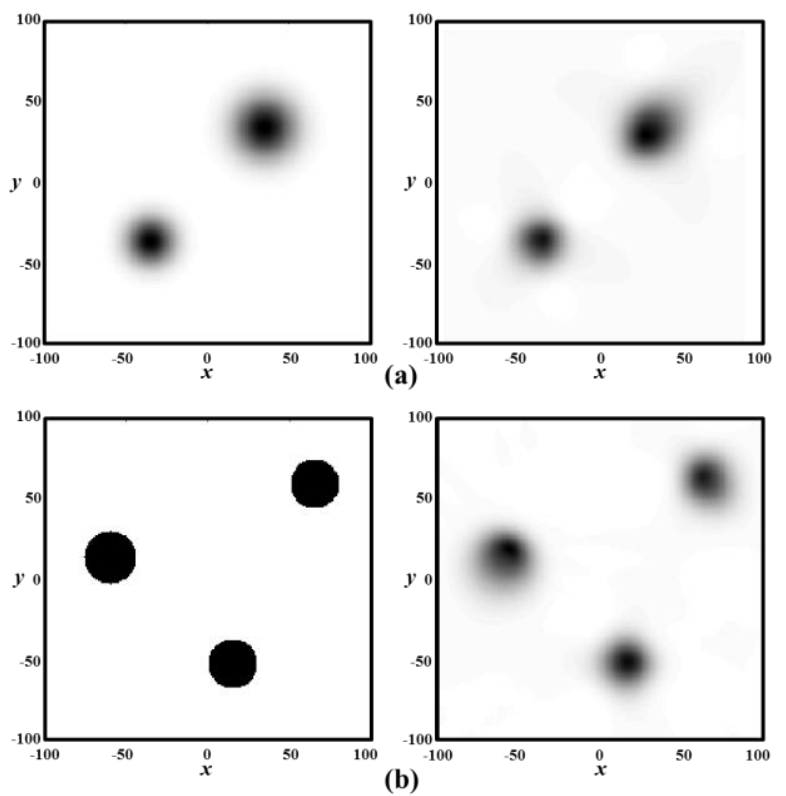

Fig. 3. Reconstruction of two phantoms (top and bottom rows) after applying parallelised versions of the SRA. The structure of the composite phantoms is shown on the left; reconstructions are shown on the right. (a) smooth edges, (b) sharp edges. 
These results validate the deployment of concurrent processing; however, this approach uses intense computational post-processing to restore the sinogram. Moreover, the need to use FBP for the reconstruction of the final image increases significantly the computational demand and potentially presents a bottleneck for real-time applications. Therefore, as mentioned in Section II, considering that for a substantial amount of applications in IPT the location of the CoMs identifies adequately the activity in the imaging frame over time, we use the alternative method based on the CoM coordinates only. This approach is supported by the relationship between the RT and HT [15], whereby the location of a peak in the $x-y$ Hough space represents the location of a CoM in the x-y image plane; hence, the SHT is indeed mapping a sinusoid back to a set of lines that intersect in the single point given by the coordinates of the relevant constituent of the SUT.

The approach we present reduces the computational cost of the original SRA further by calculating intersecting points only, as described in the next section.

\section{ALGORITHM FOR REAL-TIME APPLICATIONS}

Based on the sequential SRA, the new PCoMA was designed to reduce the computational complexity and achieve a high level of parallelism, aiming at real-time applications for tomography sensors. Given the parallelism required and the target of an embedded system, PCoMA was devised to be implemented into a FPGA board.

\section{A. Reduction to CoM form}

Coarse grain parallelism is achieved by evaluating each angular projection independently and simultaneously. In the case of four angles, it allows a real $4 \mathrm{x}$ improvement in the reduction to CoM form; this is possible because independent logic is used to process each projection, thus no communication overhead is needed. The parallel model used to describe our approach also falls within the Single Instruction Multiple Data classification, because independent components are instantiated to process every single instruction at a time on different pieces of data.

To implement the interpolation required prior to reduction to CoM form [9], each dataset representing a separate angular projection is populated concurrently, by linear spline interpolation [2], on independent logic instantiated into the FPGA fabric. The interpolated values in each angular projection are calculated between every two consecutive points according to:

$$
Y_{\theta_{i}}[k]=\left(P_{\theta_{i}}[q+1]-P_{\theta_{i}}[q]\right) \alpha[j]+P_{\theta_{i}}[q]
$$

where $q$ represents the discrete index for dataset $P_{\theta i} ; k$ is the index in the interpolated projection $Y_{\theta i}$, and $j$ enumerates the elements in the array of interpolating factors $\alpha$, which define an increment $\Delta$ equal to $1 /$ (new sampling rate); e.g. 0.250 if new sampling rate $=4$, corresponding to three points interpolated between measured data. This array is preloaded in a look-up table (LUT).

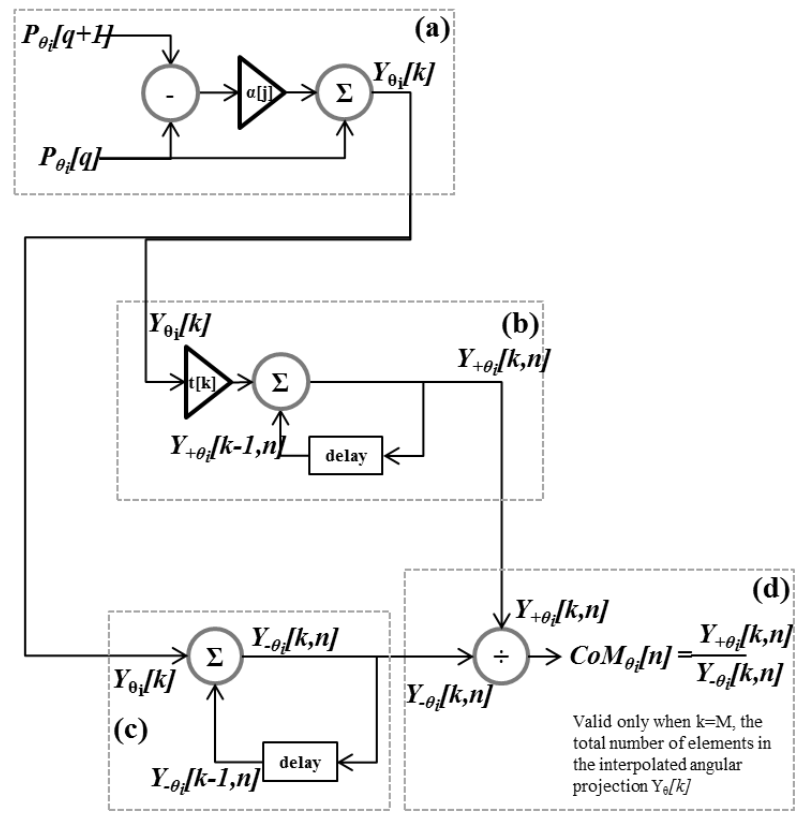

Fig. 4. Block diagram to reduce projection to CoM form; a) $Y_{\theta}[k]$ elements are obtained, b) each point is multiplied by its discrete index and added in a recursive formula generating $Y_{+\theta}[k]$, c) each $Y_{\theta}[k]$ value is also accumulated in $Y_{-\theta}[k]$ to give the total mass, d) the results of the accumulation processes are divided, the value is valid once all the elements of $Y_{\theta}[k]$ have been determined.

Additional logic is required to reduce each interpolated projection to its CoM form. To accomplish this, every time a new interpolated value is calculated it is multiplied (using independent logic) by its index $t[k, n]$ as shown in (4); then, it is added to the previous calculated value, implementing the recursive formula expressed as

$$
Y_{+\theta_{i}}[k, n]=Y_{\theta_{i}}[k, n] t[k, n]+Y_{+\theta_{i}}[k-1, n]
$$

where $k$ enumerates the elements in the interpolated projection for angle $\theta_{i}, Y_{\theta i}[k, n]$ represents every new value calculated in (3) within the bounds of the $n$th possible object, and $t[k, n]$ enumerates the discrete components of the displacement array $t$ for object $n$. Once all the points in $Y_{\theta i}[k]$ have been calculated, the CoM for all constituent objects in projection $\theta_{i}$ can be computed concurrently in only three additional operations, starting with (4). The total mass is calculated in the same manner, using an independent adder to sum every $Y_{\theta i}[k, n]$ value as soon as it is determined, according to

$$
Y_{-\theta_{i}}[k, n]=Y_{\theta_{i}}[k, n]+Y_{-\theta_{i}}[k-1, n],
$$

Finally the CoM reduction is achieved by implementing (1) in its discrete version, for each possible object found in the angular projection:

$$
\operatorname{CoM}_{\theta_{i}}[n]=\frac{Y_{+\theta_{i}}[k, n]}{Y_{-\theta_{i}}[k, n]} \leftrightarrow k=M,
$$

where $M$ represents the total number of elements in the interpolated array $Y_{\theta}[k]$. It should be noted that in order to 
guarantee the correctness of the result obtained in (6), all the interpolated points must have been calculated (see Fig. 4).

The complete process to reduce each projection to its $\mathrm{CoM}$ form is depicted in Fig. 4.

\section{B. Sinusoidal Hough Transform}

A novel approach for computing SHT is proposed in this section. In this work we consider a limited views IPT sensor, sampling only 4 angular projections at $0^{\circ}, 45^{\circ}, 90^{\circ}$ and $135^{\circ}$. For IPT applications with sparse angular data, the memory needed can be reduced significantly with the help of LUTs. Firstly, required trigonometric functions values are calculated and stored. Additional resource efficiency is achieved by employing symmetry properties: $\sin 45=\sin 135=\cos 45=$ cos 135, hence only one value is required for storage. Therefore (2) is reduced to:

$$
y=\left\{\begin{array}{l}
\frac{t_{n}}{\sin \theta}-x, \theta=45^{\circ} \\
\frac{t_{n}}{\sin \theta}+x, \theta=135^{\circ}
\end{array},\right.
$$

where $t_{n}$ represents the RT beam displacement for each $n$th constituent object at angle $\theta=45^{\circ}$ or $\theta=135^{\circ}$, i.e. the CoMform value calculated in (6).

The accumulator is then represented by a set of intersecting straight lines at $0^{\circ}, 45^{\circ}, 90^{\circ}$ and $135^{\circ}$, as shown in Fig. 5. The number of lines for every angle depends on the number of CoMs found in each projection.

The intersecting points for lines at $45^{\circ}$ and $135^{\circ}$ with those at $0^{\circ}$ are described by the discrete version of (7), given by:

$$
\begin{gathered}
y_{45}\left[n_{45}, n_{0}\right]=\frac{\operatorname{CoM}_{45^{\circ}\left[n_{45}\right]}-\operatorname{CoM}_{0^{\circ}}\left[n_{0}\right],}{\sin 45}, \\
y_{135}\left[n_{135}, n_{0}\right]=\frac{\operatorname{CoM}_{135^{\circ}\left[n_{135}\right]}^{\sin 45}+\operatorname{CoM}_{0^{\circ}}\left[n_{0}\right],}{},
\end{gathered}
$$

where $t_{n}$ has been replaced by $\operatorname{CoM}_{\theta}[n]$ obtained in (6); $n_{0}, n_{45}$ and $n_{135}$ enumerate the CoM-form objects found in projections at $0^{\circ}, 45^{\circ}$ and $135^{\circ}$ respectively; and $n_{0}, n_{45}, n_{135} \in[1, N]$ with $\mathrm{N}$ representing the total number of objects found. Notice that $\sin 45$ is used instead of $\sin 135$ in (9).

Intersecting points with those lines at $90^{\circ}$ are calculated similarly; however, in this case the intersections are with horizontal lines therefore, equations are implemented to find the $x$ coordinate as opposed to the $y$ crossing, as shown in

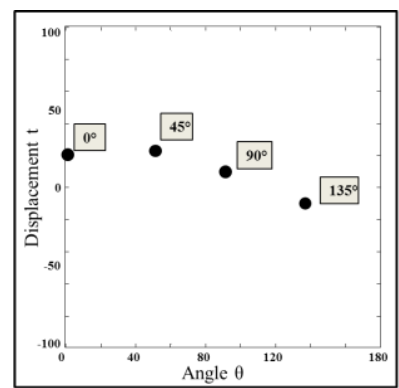

(a)

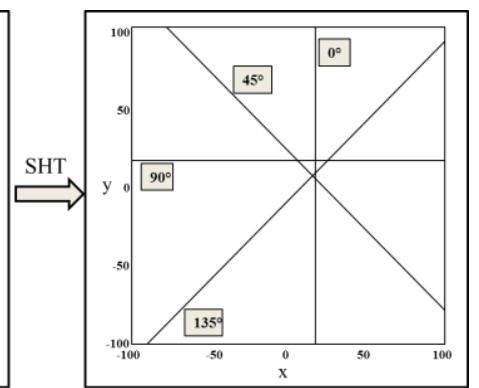

(b)
Fig. 5. a) CoM form of projections is converted to b) Hough space after applying SHT.
(10).

$$
x=\left\{\begin{array}{l}
\frac{t_{n}}{\cos \theta}-y, \theta=45^{\circ} \\
\frac{t_{n}}{\cos \theta}+y, \theta=135^{\circ}
\end{array},\right.
$$

The discrete versions of (10) are given in:

$$
\begin{gathered}
x_{45}\left[n_{45}, n_{90}\right]=\frac{\operatorname{CoM}_{45^{\circ}}\left[n_{45}\right]}{\sin 45}-\operatorname{CoM}_{90^{\circ}}\left[n_{90}\right], \\
x_{135}\left[n_{135}, n_{90}\right]=-\frac{\operatorname{CoM}_{135^{\circ}}\left[n_{135}\right]}{\sin 45}+\operatorname{CoM}_{90^{\circ}}\left[n_{90}\right],
\end{gathered}
$$

where $t_{n}$ has been replaced by $\operatorname{CoM}_{\theta}[n]$ calculated in (6); $n_{45}$, $n_{90}$, and $n_{135}$ enumerate the CoMs found in projections at $45^{\circ}$, $90^{\circ}$ and $135^{\circ}$ respectively; $n_{45}, n_{90}, n_{135} \in[1, N] ; \cos 135=-$ $\sin 45$ is used in (12).

It is important to note, that parallelism is kept a top priority in this design. For this, independent logic is used to carry out each operation in (8), (9), (11) and (12). Therefore, the time required for calculating each intersecting point is the same as that required for all the remaining intersections, as illustrated in Fig. 6.

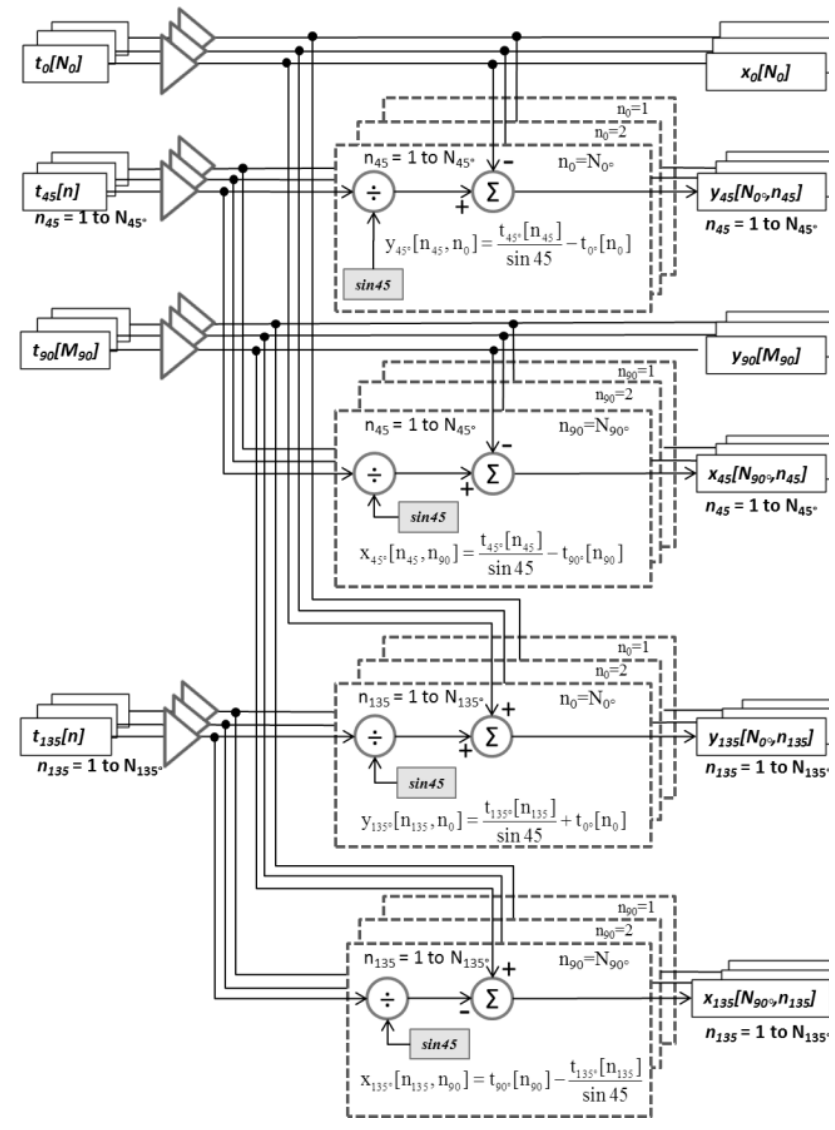

Fig. 6. Diagram showing the parallel implementation of the SHT in PCoMA: every intersection is computed simultaneously. Note that $\mathrm{M}_{0^{\circ}}$ and $\mathrm{M}_{90^{\circ}}$ represent the total number of identified objects in projections at $0^{\circ}$ and $90^{\circ}$ respectively and $\mathrm{N}_{45^{\circ}}$ and $\mathrm{N}_{135^{\circ}}$ indicate those objects found in the projections at $45^{\circ}$ and $135^{\circ}$. To make the diagram compact $t_{\theta}[n]$, $t_{\theta}[\mathrm{m}]$ are used instead of $\operatorname{CoM}_{\theta}[n]$ and $\operatorname{CoM}_{\theta}[\mathrm{m}]$. The independent equations for each intersection at $45^{\circ}$ and $135^{\circ}$ are not represented explicitly and all calculations are simultaneous. 


\section{Detection of peaks in the accumulator}

The process to identify the dominant objects of the scene uses an accumulator in the form of a matrix. The objects' locations (bins) are defined by the intersections of the lines at $0^{\circ}$ and $90^{\circ}$, i.e. the intersections of the results obtained from (6) for $\theta=0^{\circ}\left(\mathrm{CoM}_{0^{\circ}}\left[n_{0}\right]\right)$ and $\theta=90^{\circ}\left(\mathrm{CoM}_{90^{\circ}}\left[n_{90}\right]\right)$. The number $n_{90}$ of rows and $n_{0}$ of columns depends on the number of objects found in the respective projection. Note that it is not necessarily a square matrix.

To account for the error in the intersection of four lines in the accumulator, yielding the CoM coordinates of the dominant objects, we define a circular intersection bin with radius corresponding to the sampling rate (i.e. number of interpolated points between the measurements). Furthermore, a user-defined parameter $\lambda$ allows additional control on the extension of the bins; by experimentation it was determined that the optimum results are obtained when $\lambda \epsilon[1,1.5]$. Fig. 7 illustrates the accumulator bins.

Peak candidates in the accumulator are identified by a voting process which increments each bin count by 1 unit for every intersecting point, obtained from (8), (9), (11), (12), lying within the bin. Then, the bins with more counts than a predefined global threshold represent the CoMs' coordinates of the most prominent objects. The value of the threshold is predefined by the number of angular projections used, i.e. 4 in this project. However, a user-defined parameter $\tau \in(0,1]$ can be used to provide further flexibility.

Each location in the accumulator is processed concurrently, as shown in Fig. 8. The process to calculate the height of each individual bin requires only one clock cycle to be completed; therefore, all the dominant peaks in the accumulator are identified within one clock cycle once the intersections are computed.

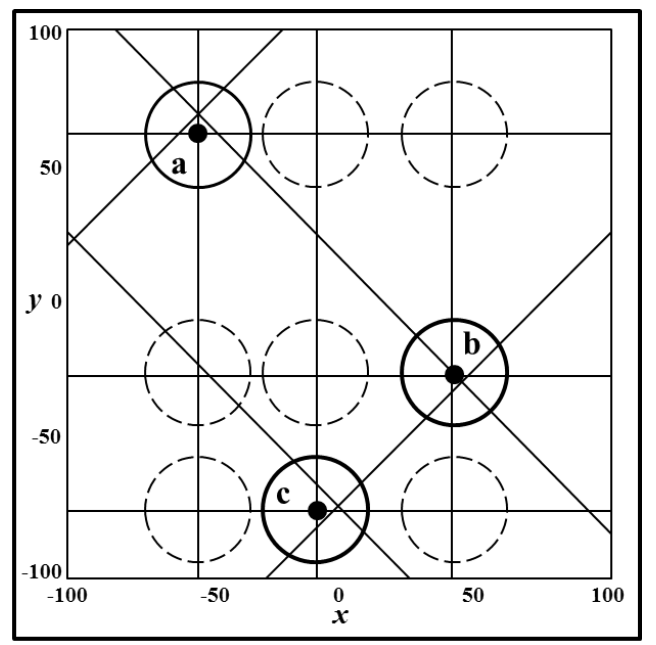

Fig. 7. Lines intersecting at $0^{\circ}$ and $90^{\circ}$ generate circular bins. In this example, nine possible bins are identified; however, only locations a, b and c cover enough intersecting points to satisfy the accumulator threshold. Hence, only these points identify dominant peaks. Their spread regions extend over a radius determined by the product of the sampling period times a factor $\lambda \in[1,1.5]$. Note that is image is just for illustration purposes and does not represent actual data.

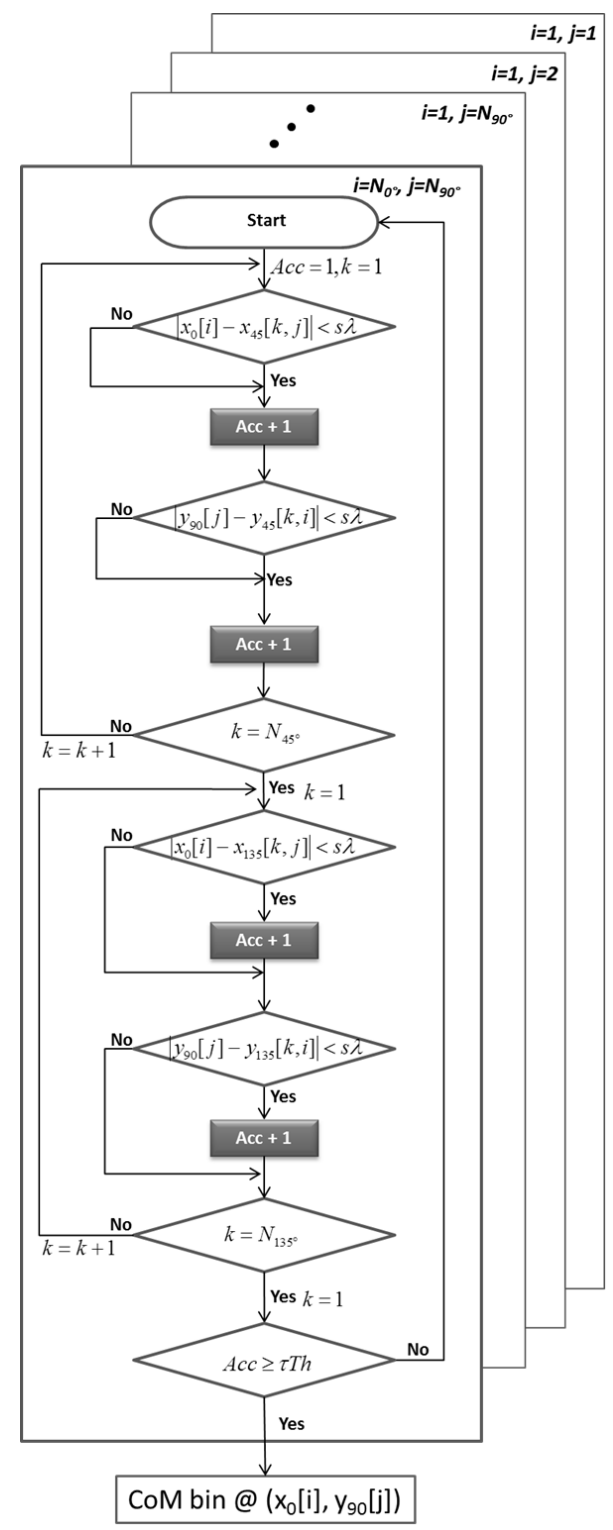

Fig. 8. Each accumulator bin is processed independently by comparing if the required number $(\tau T h)$ of crossing coordinates fall within the bin's limits given by $s \lambda$. Where $s=$ Sampling period, $\lambda=$ User-defined tolerance factor, $\mathrm{Th}=$ Accumulator bin's threshold (4 in our application),

\section{FPGA RESULTS}

The presented PCoMA was designed with the aim to be implemented on FPGA hardware. For this, the Xilinx ISE Design Suite 13.2 was used as the development environment and PCoMA was coded in Very High Speed Integrated Circuits Hardware Description Language (VHDL). Floating point operations were performed by instantiating cores with Core Generator 5.0. The algorithm was loaded on a Xilinx ML605 board with a Virtex 6 xc6vlx240t-1ff1156 FPGA.

The performance of PCoMA was evaluated with the help of suitable phantoms. Because sinogram recovery is not the main objective, phantoms with sharp edges do not pose a separate challenge in these experiments. However, for completeness, both sharp and smooth edge phantoms with 1,2 or 3 constituent objects were generated in MATLAB on a mesh of $200 \times 200$ pixels labeled from -100 to 99 with details shown in 
Table I. Their RT was calculated in MATLAB assuming 4 angular projections at $0^{\circ}, 45^{\circ}, 90^{\circ}$ and $135^{\circ}$ with 25 line integrals per projection. The RT of each phantom was loaded into FPGA memory locations and the PCoMA was run. The resultant CoM coordinates were compared to those obtained from the sequential SRA in MATLAB. The SRA computations in MATLAB were carried out on generic hardware equipped with Intel Core 2 Quad @ 2.83GHz and 4 GB of RAM. The clock used with the FPGA runs @ $66 \mathrm{MHz}$.

Table I presents the results obtained from both, the sequential SRA in MATLAB and the implementation on the Virtex 6 board. The errors from both algorithms were also calculated and compared. The error for the SRA varied between $0.02 \%$ and $5.05 \%$ for the magnitude and between negligible to $11.8 \%$ for the angle (error averages for all 10 CoMs $2.39 \%$ and $3.63 \%$ for the magnitude an angle respectively). For the PCoMA the errors varied between negligible and $2.86 \%$ for the magnitude and between negligible to $3.3 \%$ for the angle (error averages for all 10 CoMs $1.46 \%$ and $1.00 \%$ for the magnitude an angle respectively).

The results show that the coordinates calculated from the VHDL implementation match with those obtained by the MATLAB calculations. The errors are within the expected range, taking into account the sparse nature of the tomography technique employed, which is limited both radially and angularly. Furthermore, the errors are of the same order in both implementations, and in most instances marginally better for PCoMA. This can be explained due to floating point operations performed across the entire parallel algorithm, without the need for rounding of intermediate results to access elements in indexed matrices, as would be the case in the sequential version in MATLAB. The sharpness of the edges has no impact, as expected.

Table II shows that in the example cases the introduced parallelism and the transfer to the FPGA platform results in a significant improvement of at least $\times 2000$ in the processing time to calculate the CoMs' coordinates of a single object and correspondingly higher for 2 and 3 objects. As expected, the execution time of the SRA in MATLAB depends on the number of objects to process: Table II shows that the processing of phantoms with two objects takes longer than double the execution time for a single object, whilst the processing for phantoms with three objects takes more than three times that with only one. It should be noted that Table III reports only the time to calculate the CoMs' coordinates, i.e. the time to restore the sinogram is excluded.

The execution time for the FPGA board was measured using Xilinx iSim. As expected, the number of objects does not affect the execution time, which is generally very low.

\section{SUMMARY DISCUSSION AND CONCLUSIONS}

The need for a system capable of performing real-time imaging for IPT applications has been argued in preceding sections. It has also been emphasized that tomography subjects in industrial environments are often under-sampled both in the radial and angular sense.

The earlier introduced SRA is a suitable starting point to address both issues at once. However, that algorithm presents some limitations in terms of computational cost and execution time, limiting its real-time applicability. Based on prevailing scenarios in which IPT sensors are used, we assume in this work that the CoMs' coordinates of the main constituents of the SUT would yield information adequate for the correct identification of the dynamics of the scene, without performing a computationally expensive sinogram restoration.

The high parallelism of the new PCoMA algorithm makes it desirable for a FPGA implementation. In that case the execution time is significantly reduced, by at least 3 orders of magnitude, due to fine grained parallelism.

From the experiments and results presented, it can be seen that in addition to the drastic improvement in execution time, PCoMA also reduces the overall error in the results.

Whereas a considerable execution time improvement is expected in a parallel system, in our case this is also due to PCoMA executing operations on the relevant data only, i.e. only on non-zero pixels that represent useful information in the Hough space. The binning approach for accumulator processing also reduces significantly the complexity of the algorithm and achieves further parallelism.

From the results obtained, it can be concluded that the algorithm is amenable to implementation in a real-time

TABLE I

COMPARISON OF COM POSITIONS CALCULATED BY SRA IN MATLAB AND VHDL ON FPGA

\begin{tabular}{|c|c|c|c|c|c|c|c|c|c|c|c|}
\hline & & \multicolumn{2}{|c|}{ Original phantoms } & \multicolumn{4}{|c|}{ SRA MATLAB } & \multicolumn{4}{|c|}{ VHDL on FPGA } \\
\hline & & Mag & $\theta$ & Mag & $\theta$ & Err. Mag & Err. $\theta$ & Mag & $\theta$ & Err. Mag & Err. $\theta$ \\
\hline Phantom1 & CoM1 & 51.48 & -29.06 & 52 & -26 & $1.01 \%$ & $10.5 \%$ & 51.00 & -28.1 & $0.93 \%$ & $3.3 \%$ \\
\hline \multirow{2}{*}{ Phantom2 } & CoM1 & 49.50 & 45.00 & 48 & 43 & $3.03 \%$ & $4.4 \%$ & 50.91 & 45.0 & $2.84 \%$ & negligible \\
\hline & CoM2 & 49.50 & -135.00 & 47 & -135 & $5.05 \%$ & negligible & 49.50 & -135.0 & negligible & negligible \\
\hline \multirow{2}{*}{ Phantom3 } & CoM1 & 57.01 & -52.13 & 57 & -46 & $0.02 \%$ & $11.8 \%$ & 56.85 & -50.71 & $0.28 \%$ & $2.7 \%$ \\
\hline & CoM2 & 77.78 & 135.00 & 79 & 135 & $1.57 \%$ & negligible & 77.10 & 133.40 & $0.87 \%$ & $1.2 \%$ \\
\hline \multirow{2}{*}{ Phantom4 } & CoM1 & 49.5 & 45.00 & 48 & 45 & $3.03 \%$ & negligible & 50.91 & 45.0 & $2.84 \%$ & negligible \\
\hline & CoM2 & 49.5 & -135.00 & 47 & -135 & $5.05 \%$ & negligible & 48.08 & -135.0 & $2.86 \%$ & Negligible \\
\hline \multirow{3}{*}{ Phantom5 } & CoM1 & 88.46 & 42.71 & 90 & 45 & $1.74 \%$ & $5.4 \%$ & 89.87 & 42.75 & $1.60 \%$ & $0.09 \%$ \\
\hline & CoM2 & 61.85 & 165.96 & 60 & 162 & $2.99 \%$ & $2.4 \%$ & 61.13 & 164.83 & $1.16 \%$ & $0.7 \%$ \\
\hline & CoM3 & 52.20 & -73.30 & 52 & -72 & $0.39 \%$ & $1.8 \%$ & 51.55 & -71.92 & $1.24 \%$ & $1.9 \%$ \\
\hline
\end{tabular}

Note that Phantom1 consist of a single object; Phantom 2 and Phantom 4 differ in that the latter has smooth edges; Phantom 5 comprises 3 objects 
TABLE II

TIME EXECUTION COMPARISON

\begin{tabular}{|c|c|c|c|}
\cline { 2 - 4 } \multicolumn{1}{c|}{} & \multicolumn{2}{c|}{ Time execution (seconds) } & \multicolumn{1}{c|}{} \\
\cline { 2 - 4 } \multicolumn{1}{c|}{} & MATLAB & VHDL & Improvement \\
\hline Phantom1 & 0.05400 & $26.00 \mathrm{E}-06$ & $\approx 2076 \mathrm{x}$ \\
\hline Phantom2 & 0.14306 & $26.00 \mathrm{E}-06$ & $\approx 5502 \mathrm{x}$ \\
\hline Phantom3 & 0.13704 & $26.00 \mathrm{E}-06$ & $\approx 5271 \mathrm{x}$ \\
\hline Phantom4 & 0.14189 & $26.00 \mathrm{E}-06$ & $\approx 5457 \mathrm{x}$ \\
\hline Phantom5 & 0.32404 & $26.00 \mathrm{E}-06$ & $\approx 12463 \mathrm{x}$ \\
\hline
\end{tabular}

system. It should be noted that only $37 \%$ of the slices and $26 \%$ of the DSP logic available in the selected FPGA were used, leaving enough resources to implement a data acquisition system in the same board, leading to a fully embedded tomography system with less resources than the hereby demonstrated. Furthermore, the modular design of PCoMA allows modifications to the sensor geometry without incurring major changes to the developed code; upgrades of the number of line integrals or angular projections should be indeed seamless.

Tomography sensing applications would benefit from embedded systems across a number of modalities [16]. Obvious candidates are Photonic Guided-path Tomography (PGPT) [17] and Hard-Field THz Tomography [18], where CoM information can be the ultimate target for e.g. smart carpets [17] or temperature distribution tracking [19]. However, it should be noted that the full potential of PCoMA can be achieved only with tomography sensors allowing true parallel data acquisition from a large number of measurement channels [10], possibly integrated on the same FPGA chip.

\section{REFERENCES}

[1] M. I. Sezan and H. Stark, "Tomographic Image Reconstruction from Incomplete View Data by Convex Projections and Direct Fourier Inversion," IEEE Transactions on Medical Imaging, vol. 3, pp. 91-98, 1984.

[2] J. L. Prince and A. S. Willsky, "Hierarchical reconstruction using geometry and sinogram restoration," IEEE Transactions on Image Processing, vol. 2, pp. 401-416, 1993.

[3] A. H. Andersen, "Algebraic reconstruction in CT from limited views," IEEE Transactions on Medical Imaging, vol. 8, pp. 50-55, 1989.

[4] B. P. Medoff, W. R. Brody, M. Nassi, and A. Macovski, "Iterative convolution backprojection algorithms for image reconstruction from limited data," J. Opt. Soc. Am., vol. 73, pp. 1493-1500, 1983.

[5] N. Terzija and H. McCann, "Wavelet-Based Image Reconstruction for Hard-Field Tomography With Severely Limited Data," IEEE Sensors Journal, vol. 11, pp. 1885-1893, 2011.

[6] E. P. A. Constantino and K. B. Ozanyan, "Tomographic Imaging of Surface Deformation From Scarce Measurements via Sinogram Recovery," IEEE Sensors Journal, vol. 9, pp. 399-410, 2009.

[7] N. Nurgiyatna, P. Scully, and K. B. Ozanyan, "Grooved fiber sensors for deformation imaging," in Proc. IEEE SENSORS 2010, pp. 1675-1680, 2010.

[8] E. Constantino and K. Ozanyan, "Sinogram recovery for sparse angle tomography using a sinusoidal Hough transform," Measurement Science and Technology, vol. 19, p. 11, 2008.

[9] K. B. Ozanyan, S. G. Castillo, and F. J. P. Ortiz, "Guided-path tomography sensors for nonplanar mapping," IEEE Sensors Journal, vol. 5, pp. 167-174, 2005.

[10] S. G. Castillo and K. B. Ozanyan, "Field-programmable data acquisition and processing channel for optical tomography systems," Review of Scientific Instruments, vol. 76, pp. 095109-095109-6, 2005.
[11] C. Guerra and S. Hambrusch, "Parallel algorithms for line detection on a mesh," Journal of Parallel and Distributed Computing, vol. 6, pp. 1-19, 1989.

[12] S. S. Sathyanarayana, R. K. Satzoda, and T. Srikanthan, "Exploiting Inherent Parallelisms for Accelerating Linear Hough Transform," IEEE Transactions on Image Processing, vol. 18, pp. 2255-2264, 2009.

[13] C. Ling, C. Hongjian, P. Yi, and C. Yixin, "A Fast Efficient Parallel Hough Transform Algorithm on LARPBS," J. Supercomput., vol. 29, pp. 185-195, 2004.

[14] C. Yen-Kuang, L. Wenlong, L. Jianguo, and W. Tao, "Novel parallel Hough Transform on multi-core processors," Proc. IEEE International Conference on Acoustics, Speech and Signal Processing, 2008. ICASSP, pp. 1457-1460, 2008.

[15] S. R. Deans, "Hough Transform from the Radon Transform," IEEE Transactions on Pattern Analysis and Machine Intelligence, vol. PAMI3, pp. 185-188, 1981.

[16] T. York, H. McCann, and K. B. Ozanyan, "Agile Sensing Systems for Tomography," IEEE Sensors Journal, vol. 11, pp. 3086-3105, 2011.

[17] J. Cantoral Ceballos, N. Nurgiyatna, P. Scully, and K. B. Ozanyan, "Smart carpet for imaging of objects' footprint by photonic guided-path tomography," Proc. AFRICON 2011, pp. 1-6, 2011,

[18] K. B. Ozanyan, P. Wright, M. R. Stringer, and R. E. Miles, "Hard-Field THz Tomography," IEEE Sensors Journal, vol. 11, pp. 2507-2513, 2011.

[19] M. Wood and K. Ozanyan, "Temperature Imaging with Molecular Absorption Tomography Sensors," IEEE Sensors Journal 2012, submitted.

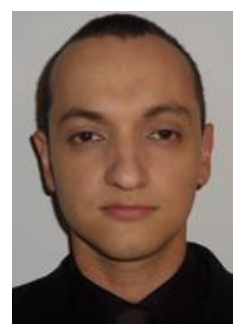

Jose A. Cantoral-Ceballos (S'10) received the B.Sc. degree (Excellence Hons) in Electronic and Communications Engineering from ITESM, Queretaro, Mexico and the M.Sc. (Distinction) in Sensors and Electronic Instrumentation from The University of Manchester, Manchester, U.K., in 2005 and 2009, respectively. He was awarded the National Instruments prize in 2009 for his M.Sc Dissertation.

From 2005 to 2008 he was with CIATEQ A.C., Mexico, as a Project Engineer in the development of SCADA systems for the oil and gas industry. He is currently pursuing the $\mathrm{Ph} . \mathrm{D}$. degree working on parallel algorithms for imaging from limited data industrial tomography at The University of Manchester.

Mr. Cantoral-Ceballos has been a student member of the Institute of Engineering and Technology (IET, formerly IEE) since 2010.

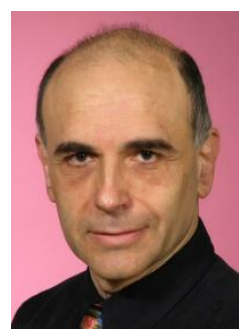

Krikor B. Ozanyan (M'1995, SM'2003) received his MSc degree in engineering physics (semiconductors) and $\mathrm{PhD}$ degree in solid-state physics, in 1980 and 1989 respectively, from the University of Sofia, Bulgaria.

He has held previous academic and research posts in the University of Sofia, The Norwegian Institute of Technology (Trondheim, Norway), the University of Hull (UK), and the University of Sheffield (UK), working on projects ranging from Brewster-angle midIR spectroscopic ellipsometry and electron confinement in quantum wells and barriers, to the demonstration of the lasing at $333 \mathrm{~nm}$ from strained MQW $\mathrm{ZnCdS} / \mathrm{ZnS}$ structures and in-situ real-time optical monitoring of growth of III-V semiconductors in MBE and MOCVD reactors. His current interests are in the area of photonic sensors and indirect imaging (tomography) by optical modalities, signal processing for optical experiments, and spectroscopy with ultrafast laser sources. He is currently Head of Sensors, Imaging and Signal Processing at the University of Manchester and Visiting Professor at the University of Bergen, Norway.

Professor Ozanyan is Fellow of the Institute of Engineering and Technology, (UK, formerly IEE) and Fellow of the Institute of Physics (UK). He was Distinguished Lecturer of the IEEE Sensors Council in 2009-2010, Guest Editor of the IEEE Sensors Journal Special Issues "Sensors for Industrial Process Tomography" in 2005 and "THz Sensing: Materials, Devices and Systems" in 2012. He is currently Editor-in-Chief of the IEEE Sensors Journal. 